\title{
Aneurisma y fístula arterio-venosa renal: manejo quirúrgico laparoscópico asistido por robot*
}

\author{
Drs. OCTAVIO A. CASTILLO C. ${ }^{1,2}$, MARIANO CUENTAS J. ${ }^{1}$, RUBÉN VIDANGOS V. ${ }^{1}$, \\ RODRIGO GUTIÉRREZ ${ }^{1}$, PATRICIO PALAVECINO R. ${ }^{3}$
}

1 Unidad de Urología y Centro de Cirugía Robótica, Clínica INDISA.

2 Facultad de Medicina, Universidad Andrés Bello.

3 Unidad de Radiología Intervencionista, Clínica INDISA.

Santiago, Chile.

\begin{abstract}
Renal artery aneurysm. Robotic resolution

Introduction: Renal artery aneurysm is uncommon conditions, presenting a therapeutic challenge. Aim: To report a case of a complex intrarenal aneurysm associated with a arterio-venous fistula high flow, treated by robotic assistance. Case report: A 51 year-old man, with a long history of hypertension, was diagnosed of a complex intrarenal aneurysm, in routine radiological follow-up. Endovascular treatment was no possible due to an associated high flow arterio-venous, so robotic surgery was performed. The tributary arterial branch was dissected at the renal pedicle, and ligated easily. The radiological follow-up showed a complete resolution and normal renal function. Conclusion: This case, of low frequency, illustrates a successfully manner of resolution of a complex renal vascular pathology.
\end{abstract}

Key words: Aneurysm, renal artery, vascular malformation, laparoscopic surgery, robotic surgery, endovascular treatment.

\section{Resumen}

Introducción: Los aneurismas de la arteria renal constituyen una patología infrecuente, y plantean dificultades en la decisión terapéutica. Objetivo: Presentar un caso de un aneurisma complejo intrarenal, asociado a una fístula arterio-venosa de alto flujo, el cual fue resuelto con asistencia robótica. Caso clínico: Paciente de 51 años, con historia prolongada de hipertensión arterial, al cual, en estudio radiológico de rutina, se le diagnostica 3 aneurismas intrarenales derechos, asociado a una fístula arterio-venosa de alto flujo. Dada la imposibilidad de realizar tratamiento endovascular, se realiza cirugía robótica. Se diseca el hilio renal y se identifica la rama arterial tributaria del aneurisma, la cual se liga sin problemas. El control alejado demuestra resolución de la malformación, con mantención de la función renal. Conclusión: Este caso, de baja frecuencia, ilustra una forma de resolución de una patología vascular renal, con éxito.

Palabras clave: Aneurisma, arteria renal, malformación vascular renal, cirugía laparoscópica, cirugía robótica, tratamiento endovascular.

*Recibido el 19 de marzo de 2013 y aceptado para publicación el 27 de mayo de 2013.

Conflicto de interés: Dr. Octavio A. Castillo C. es Proctor para Latinoamérica del robot da Vinci (Intuitive Surgical Inc.).

Correspondencia: Dr. Octavio A. Castillo C.

Avenida Santa María 1810, Santiago, Chile. P.C.: 7520440

octavio.castillo@indisa.cl 


\section{Introducción}

Los aneurismas de la arteria renal son dilataciones localizadas de la misma o de sus ramas, con una incidencia que varía entre 0,4 y $1,32 \%$ de la población general ${ }^{1,2}$. En su mayoría son asintomáticos, siendo diagnosticados en controles rutinarios por hipertensión arterial o como hallazgo incidental en estudios imagenológicos ${ }^{3-5}$. La asociación entre aneurisma renal y fístula arterio-venosa es aún más infrecuente, con pocos casos documentados en la literatura en riñones nativos ${ }^{6}$.

La importancia radica en el riesgo de rotura espontánea, el cual se incrementa cuando el diámetro excede $1,5 \mathrm{~cm}$; si, en mujeres gestantes puede asociarse a un sangrado masivo aún con diámetros menores a $1,5 \mathrm{~cm}^{7-12}$.

El tratamiento de los aneurismas de la arteria renal comprende desde técnicas endovasculares a variadas reparaciones quirúrgicas, dependiendo de su localización, características anatómicas y la experiencia del equipo quirúrgico.

Este caso ilustra el hallazgo de una aneurisma de arteria renal asociado a fístula arterio-venosa, de diagnóstico incidental y de resolución con cirugía robótica.

\section{Caso clínico}

Paciente varón de 51 años de edad, con antecedentes clínicos de hipertensión arterial de 20 años de evolución. Como parte de un estudio clínico de rutina se realiza una Tomografía computada de abdomen que revela la presencia de una malformación vascular compatible con dilataciones aneurismáticas intrarenales, la mayor de $4,2 \mathrm{~cm}$, localizadas en cara anterior del tercio medio del riñón derecho (Figura 1). Se le realizó una angiografía selectiva renal derecha que demuestra la presencia de 3 formaciones saculares intrarenales con afluente arterial de una rama importante de la arteria renal principal, de 8 $\mathrm{mm}$ (Figura 2) y la presencia de una gran fistula arterio venosa que llena rápidamente la vena renal principal.

Dado el riesgo de embolización accidental de los elementos de oclusión endovascular, por la presencia de una fístula arterio-venosa de alto flujo, se decide realizar ligadura selectiva de la arteria afluente de la malformación renal vascular.

Para ello se utiliza el sistema robótico da Vinci S-HD (Intuitive Surgical, Sunny Valley, California, USA). Se identifica el pedículo renal y se identifica la arteria renal derecha principal y la rama afluente del aneurisma (Figura 3). Se procede a la ligadura selectiva de esta rama arterial, sin incidentes (Figu-

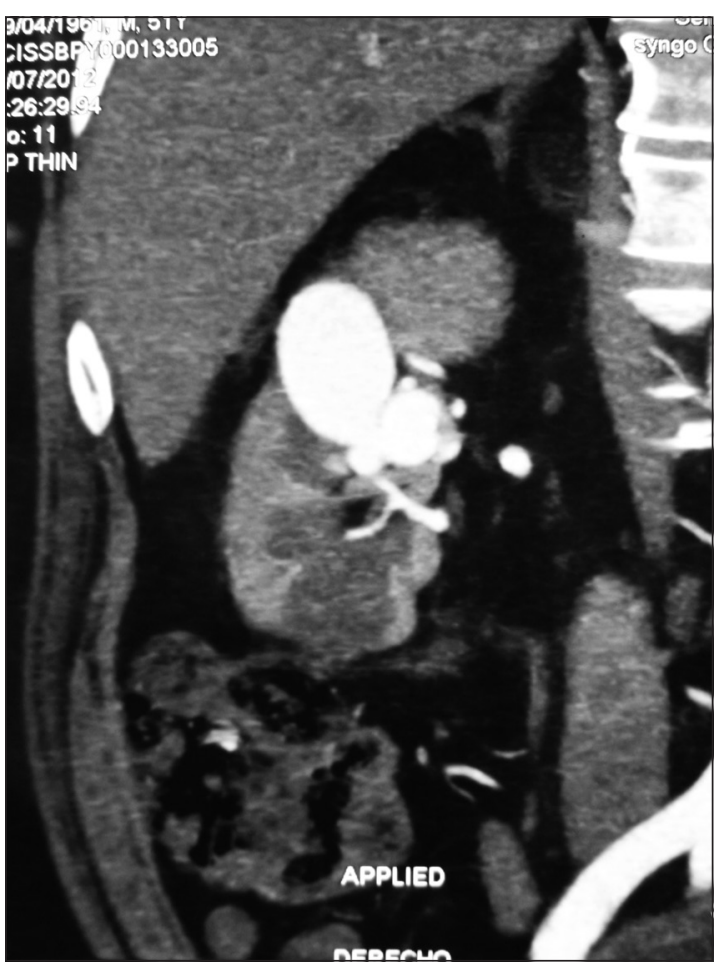

Figura 1. Tomografía computada que muestra dilataciones aneurismáticas intra-renales, la mayor de $4,2 \mathrm{~cm}$, en el tercio medio del riñón derecho.

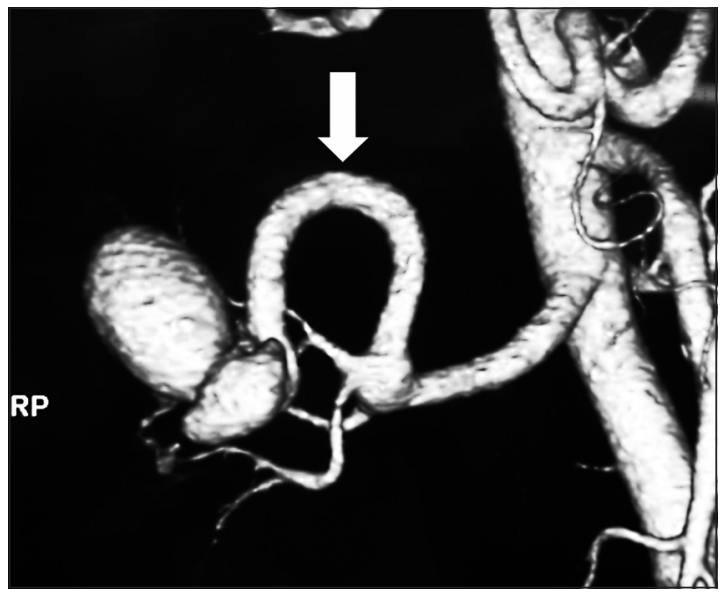

Figura 2. Reconstrucción tridimensional de angiografía renal derecha que muestra 3 formaciones saculares con afluente arterial desde la arteria renal principal, de $8 \mathrm{~mm}$ (Flecha).

ra 4). El paciente es dado de alta a las $48 \mathrm{~h}$ y una tomografía computada de control muestra ausencia de aneurismas y fístula arterio-venosa, con buena función y perfusión renal derecha (Figura 5). 


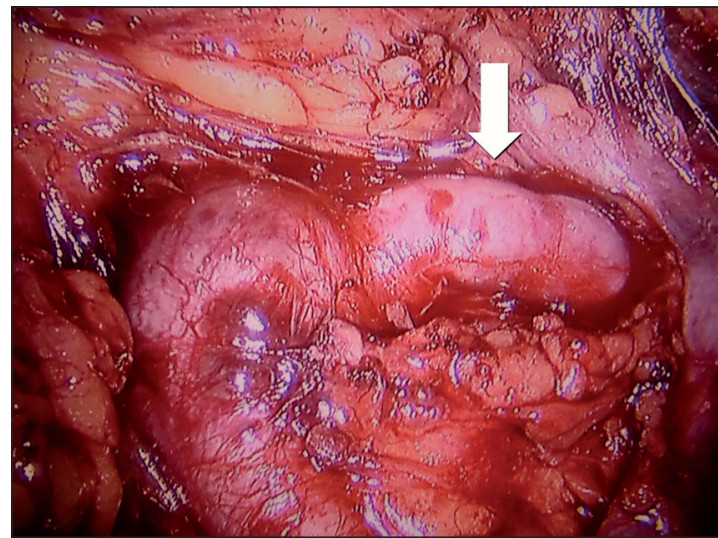

Figura 3. Disección del pedículo renal con identificación de la arteria renal derecha principal y la rama afluente del aneurisma (Flecha).

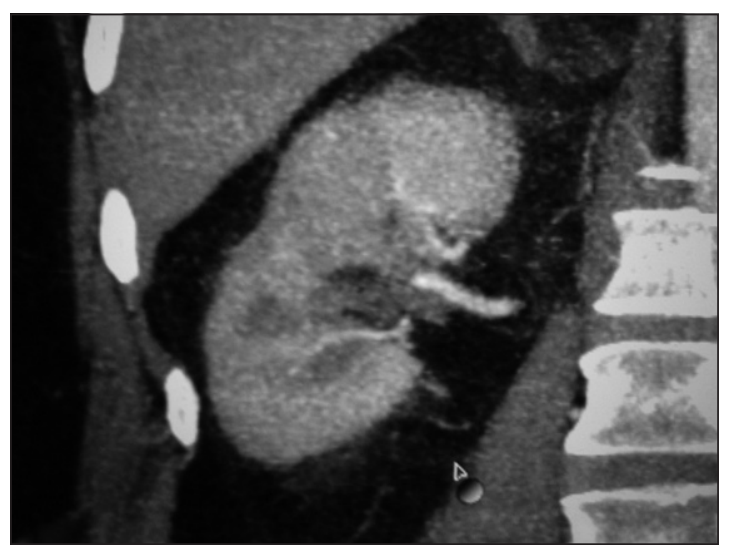

Figura 5. Tomografía computada de control con resolución de la malformación y sin compromiso vascular del parénquima renal (Figura 5).

\section{Discusión}

Los aneurismas de la arteria renal son muy infrecuentes y su etiología suele estar en relación con displasia fibromuscular o arteriosclerosis de la arteria renal, pudiendo ser congénitos, asociados con arteritis, o con antecedente traumático ${ }^{2-5}$. Según Poutasse, los aneurismas de la arteria renal se dividen en cuatro tipos: Saculares, Fusiformes, disecantes e intrarrenales ${ }^{13}$, siendo los más frecuentes los saculares que comprenden el $70 \%$ a $90 \%$ de todos los aneurismas de la arteria renal y se ubican por lo general en la bifurcación de la arteria renal principal o una de sus ramas.

Las complicaciones de los aneurismas renales incluyen trombosis con infarto renal, fistulas arteriovenosas, hipertensión renovascular y rotura espontánea que puede poner en peligro la vida del

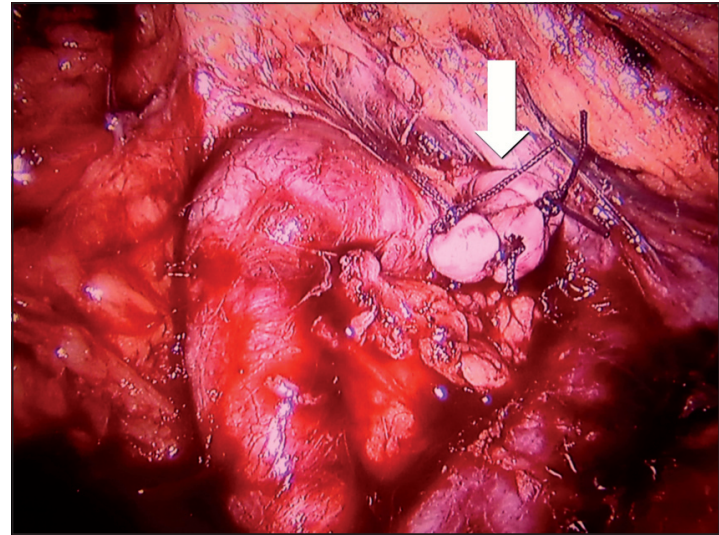

Figura 4. Ligadura selectiva de afluente arterial (Flecha).

paciente $\mathrm{e}^{7-10}$. La rotura espontánea puede ocurrir en el $5 \%$ de los aneurismas y con mayor probabilidad en los que no son calcificados o son parcialmente calcificados mayores de $1,5 \mathrm{~cm}^{8-12}$. Los factores de riesgo para rotura ocurre principalmente ante la presencia de hipertensión y en mujeres gestantes, relacionado al incremento del flujo renal ${ }^{7-13}$. El manejo conservador se puede realizar en pacientes normotensos y con aneurismas calcificados menores a $1,5 \mathrm{~cm}$. Se acepta que un aneurisma mayor de $2 \mathrm{~cm}$ requiere tratamiento quirúrgico ${ }^{8-12}$.

Las opciones de tratamiento son diversas y van a depender del tamaño y la ubicación del aneurisma. Entre las alternativas tenemos: embolización, resección del aneurisma con anastomosis términoterminal, reparación extracorpórea en cirugía de banco con autotrasplante, nefrectomía parcial y nefrectomía total. Gill et al $^{11}$, comunican el primer caso de un aneurisma renal de $3 \mathrm{~cm}$, resuelto por vía laparoscópica. Castillo et al ${ }^{12}$, comunican a su vez 2 casos de aneurisma renal, con reparación laparoscópica exitosa.

Las limitaciones de esta vía son principalmente en la dificultad de la técnica, operando en un campo quirúrgico reducido y profundo; que además, requiere precisión para una cirugía microvascular ${ }^{13,14}$.

Con la aparición de la cirugía robótica, se han ido superando las limitaciones de la cirugía laparoscópica. El robot ofrece una visión tridimensional y ampliada del campo quirúrgico, con una excelente resolución, y elimina el temblor natural de las manos del cirujano.

Luke et $\mathrm{al}^{14}$, en el año 2006, comunican un caso de reparación laparoscópica asistida por robot de un aneurisma renal izquierdo de $25 \mathrm{~mm}$, con un tiempo de isquemia de $59 \mathrm{~min}$. Guilianoti et $\mathrm{al}^{15}$, en el 2010, comunica una serie de 5 casos de reparación quirúr- 
gica laparoscópica asistida por robot, con excelentes resultados.

Nuestro caso ilustra un enfoque quirúrgico mínimamente invasivo que ha resuelto una malformación vascular renal de rara presentación en riñones nativos. Este caso carece de la dificultad de la reconstrucción vascular propiamente tal, dado que la cirugía consistió en una simple ligadura de la rama arterial aferente. Sin embargo, ilustra una malformación aneurismática intrarenal severa, con importante riesgo vital o de pérdida del riñón. La asistencia robótica facilitó la disección del hilio renal y de sus ramas, en posición intrasinusal, identificando y controlando la rama arterial responsable de la malformación, y al mismo tiempo manteniendo un riñón completamente funcionante.

\section{Referencias}

1. Tham G, Ekelund L, Herrlin K, Lindstedt EL, Olin T, Bergentz SE. Renal artery aneurysms. Natural history and prognosis. Ann Surg. 1983;197:348-52.

2. Fergany A, Novick A. Renovascular Hypertension and ischemic nephropathy En: Campbell - Walsh Urology, $10^{\mathrm{a}}$ edición, Ed. Kavoussi L, Partin A, Novick A, Peters C, Elsevier Saunders, Philadelphia, PA 19103-2899, pág. 1078-80.

3. Vaughan TJ, Barry WF, Jeffords DL, Johnsrude IS. Renal artery aneurysms and hypertension. Radiology 1971;99:287-93.

4. Henke PK, Cardneau JD, Welling TH, Upchurch GR Jr, Wakefield TW, Jacobs LA, et al. Renal artery aneurysms a 35-year clinical experience with 252 aneurysms in 168 patients. Ann Surg. 2001;234:454-63.

5. Hageman JH, Smith RF, Szilagyi E, Elliot JP. Aneurysms of the renal artery: problems of prognosis and surgical management. Surgery 1978;84:563-72.
6. Trocciola SM, Chaer RA, Lin SC, Dayal R, Scherer M, Garner M, et al. Embolization of renal artery aneurysm and arteriovenous fistula. Case report. Vasc Endovascular Surg. 2005;39:525-9.

7. Beseth BD, Quinones-Baldrich WJ. Renal artery aneurysm secondary to fibromuscular dysplasia in a young patient. Ann Vasc Surg. 2005;19:605-8.

8. Ortenberg J, Novick AC, Straffon BH, Stewart BH. Surgical treatment of renal artery aneurysms. BJU 1983; 55:341-6.

9. Lacombe M. Ex situ repair of complex renal artery aneurysms. Cardiovasc Surg. 1994;2:767-71.

10. Dubernard JM, Martin X, Gelet A, Mongin D. Aneurysms of the renal artery: surgical management with special reference to extracorporeal surgery and autotrasplantation. Eur Urol. 1985;11:26-30.

11. Gill IS, Murphy DP, Hsu TH, Fergany A, El Fettouh, Meraney AM. Laparoscopic repair of a renal artery aneurysm. J Urol. 2001;166:202-5.

12. Castillo OA, Peacock L, Díaz M, Orellana S, Urena RD. Case report: Laparoscopic Repair of Saccular RenalArtery Aneurysm. J Endourol. 2006;20:260-1.

13. Castillo OA, Vitagliano GJ, Sánchez-Salas R, Chamorro H, Fava M, Díaz MA. Laparoscopic repair of renal artery aneurysm:a description of the technique and results in 2 cases. Surg Laparosc Endosc Percutan Tech. 2008;18:379-83.

14. Poutasse EF. Renal Artery Aneuryms. Their Natural history and Surgery. Trans Am Assoc Genitourin Surg. 1979;71:146-53.

15. Luke P, Knudsen BE, Nguan CY, Pautler SE, Swinnimer S, Kiaii R, et al. Robot-assisted laparoscopic renal artery aneurysm reconstruction. J Vasc Surg. 2006;44:651-3.

16. Giulianotti PC, Bianco, FM, Addeo P, Lombardi A, Coratti A, Sbrana F. Robot-assisted laparoscopic repair of renal artery aneurysms J Vasc Surg. 2010;51:842-9. 